This item was submitted to Loughborough's Research Repository by the author.

Items in Figshare are protected by copyright, with all rights reserved, unless otherwise indicated.

\title{
Thermodynamics of a Fermi liquid in a magnetic field
}

PLEASE CITE THE PUBLISHED VERSION

http://dx.doi.org/10.1103/PhysRevB.72.115112

\section{PUBLISHER}

(C) The American Physical Society

VERSION

VoR (Version of Record)

LICENCE

CC BY-NC-ND 4.0

\section{REPOSITORY RECORD}

Betouras, Joseph J., Dmitry V. Efremov, and Andrey Chubukov. 2019. "Thermodynamics of a Fermi Liquid in a Magnetic Field". figshare. https://hdl.handle.net/2134/12775. 


\title{
Thermodynamics of a Fermi liquid in a magnetic field
}

\author{
Joseph Betouras, ${ }^{1}$ Dmitri Efremov, ${ }^{2}$ and Andrey Chubukov ${ }^{3}$ \\ ${ }^{1}$ Max-Planck-Institut für Physik Komplexer Systeme, Nöthnitzer Strasse 38, 01187 Dresden, Germany \\ ${ }^{2}$ Lehstruhl für Festkörpertheorie, TU Dresden Institut für Theoretische Physik, 01062 Dresden, Germany \\ ${ }^{3}$ Department of Physics and Condensed Matter Theory Center, University of Maryland, College Park, Maryland 20742-4111, USA
}

(Received 12 April 2005; published 14 September 2005)

\begin{abstract}
We extend previous calculations of the nonanalytic terms in the spin susceptibility $\chi_{s}(T)$ and the specific heat $C(T)$ to systems in a magnetic field. Without a field, $\chi_{s}(T)$ and $C(T) / T$ are linear in $T$ in two dimensions (2D), while in $3 \mathrm{D}, \chi_{s}(T) \propto T^{2}$ and $C(T) / T \propto T^{2} \ln T$. We show that in a magnetic field, the linear in $T$ terms in $2 \mathrm{D}$ become scaling functions of $\mu_{B} H / T$. We present explicit expressions for these functions and show that at high fields $\mu_{B} H \gg T, \chi_{s}(T, H)$ scales as $|H|$. We also show that in $3 \mathrm{D}, \chi_{s}(T, H)$ becomes nonanalytic in a field and at high fields scales as $H^{2} \ln |H|$.
\end{abstract}

DOI: 10.1103/PhysRevB.72.115112

PACS number(s): 71.10.Ay, 71.10.Pm

\section{INTRODUCTION}

Landau Fermi-liquid theory ${ }^{1}$ provides the basis for our present understanding of correlated electronic systems. The theory predicts that, in any Fermi liquid, the spin susceptibility $\chi_{s}(T)$ and the specific heat coefficient $\gamma(T)=C(T) / T$ tend to a constant at $T \rightarrow 0 .^{1,2}$ Later, Landau theory has been extended to include the leading temperature dependence of $\chi_{s}(T)$ and $\gamma(T)$ which turn out to be nonanalytic in dimensions $D \leqslant 3 \cdot^{3-14}$ As with the zero-temperature terms, the thermal corrections come from fermions in the immediate vicinity of the Fermi surface. In two-dimensional (2D) systems, both $\chi_{s}(T)$ and $\gamma(T)$ are linear in $T$ II (Refs. 7-10) and the coefficients are expressed in terms of charge and spin components of the scattering amplitude at the scattering angle $\theta=\pi .^{12,13}$ In 3D, $\chi_{s}(T)$ is quadratic in $T$, i.e., is analytic, ${ }^{4,5,6}$ while $\gamma(T)$ is nonanalytic and scales as $T^{2} \ln T^{3-5}$

In this paper, we extend previous works to systems in a magnetic field $H$. We consider $S=1 / 2$ chargeless fermions (such as ${ }^{3} \mathrm{He}$ atoms) for which the magnetic field adds a spin-dependent Zeeman term $\pm \mu_{B} H$ to the fermionic dispersion. We show that, in the presence of a field $\Delta \chi_{s}(T, H)=\chi_{s}(T, H)-\chi_{s}(0,0) \quad$ and $\quad \Delta \gamma(T, H)=\gamma(T, H)$ $-\gamma(0,0)$ become scaling functions of $\mu_{B} H / T: \Delta \chi_{s}(T, H)$ $=\Delta \chi_{s}(T, 0) f_{\chi}\left(\mu_{B} H / T\right), \Delta \gamma(T, H)=\Delta \gamma(T, 0) f_{\gamma}\left(\mu_{B} H / T\right)$. We present the expressions for these functions to second order in the interaction potential $U$ and we discuss the extension to an arbitrary interaction $U(q)$, where $q$ is the momentum transfer. For 2D systems, we show that at $\mu_{B} H \gg T$ (but still, $\mu_{B} H$ $\left.\ll E_{F}\right), \Delta \chi_{s}(T, H)$ scales as $|H|$ and weakly depends on $T$. In the same field range, $\delta \gamma(T, H)$ is still linear in $T$, but the prefactor is different from that at $H=0$. For 3D systems, we show that $\Delta \chi_{s}(T, H)$ becomes nonanalytic at a nonzero $H$. The nonanalytic term in $\Delta \chi_{s}(T, H)$ scales as $H^{2} \ln \left[\max \left(\mu_{B} H, T\right) / E_{F}\right]$. The specific heat coefficient $\gamma(H, T)$ in a field still scales as $T^{2} \ln T$, but, as in $2 \mathrm{D}$, the prefactor changes between $H=0$ and $\mu_{B} H \gg T$. Finally, we argue that the scaling behavior of $\Delta \chi_{s}(T, H)$ and $\Delta \gamma(T, H)$ is a Fermi-liquid effect, and it survives in an arbitrary Fermi liquid.

The analysis of the behavior of $\Delta \chi_{s}(T, H)$ and $\Delta \gamma(T, H)$ in a magnetic field may be useful for the experimental verifica- tions of the nonanalytic behavior of the thermodynamic variables. Our theory can be applied to ${ }^{3} \mathrm{He},{ }^{15}$ and $2 \mathrm{D}$ and 3D electronic systems, such as, e.g., GaAs/AlGaAs (Ref. 16) and Si inversion layers. ${ }^{17}$ Experimentally, it is more straightforward to analyze the dependence on the magnetic field rather than the dependence on the temperature. In particular, recent measurements of the temperature dependence of the spin susceptibility in 2D Si inversion layers ${ }^{17}$ did not yield conclusive results on whether the $T$ dependence of $\chi_{s}(T)$ is indeed linear, as some temperature dependence inevitably comes from spins on the substrate. For this and other systems, our results call for measurements of the field dependence of the spin susceptibility at a given $T$. Our scaling functions can then be used to fit the data.

\section{COMPUTATIONAL PROCEDURE}

The point of departure for our calculations is the LuttigerWard expression for the thermodynamic potential. To simplify the presentation, we assume that the interaction potential $U(q)$ is independent on $q$. We restore the momentum dependence of $U(q)$ in the final formulas. To second order in $U$, the thermodynamic potential is the sum of two closed diagrams. It can be conveniently represented by

$$
\Phi=\Phi_{0}-\frac{U^{2}}{2} T \sum_{n} \int_{q} \frac{d^{d} q}{(2 \pi)^{d}} \Pi^{\uparrow \uparrow}\left(\vec{q}, \Omega_{n}, T\right) \Pi^{\downarrow}\left(\vec{q}, \Omega_{n}, T\right),
$$

where $\Phi_{0}$ is the thermodynamic potential for free fermions and $\Pi^{\uparrow \uparrow}\left(\vec{q}, \Omega_{n}, T\right)$ and $\Pi^{\downarrow}\left(\vec{q}, \Omega_{n}, T\right)$ are the particle-hole bubbles composed of fermions with spin up or spin down, respectively.

Previous studies of the spin susceptibility and the specific heat in a zero magnetic field established that the nonanalytic temperature behavior of $\Delta \chi_{s}(T)$ and $\Delta \gamma(T)$ originates from the nonanalyticity of the polarization operator either near $q$ $=0$ (Landau damping $)^{3-5,10}$ or near $q=2 k_{F}$ (a dynamic Kohn anomaly). $6,7,9,10,13,14$ The $2 k_{F}$ nonanalyticity contributes to the spin susceptibility and the specific heat, while the $q=0$ nonanalyticity only contributes to the nonanalyticity in the 

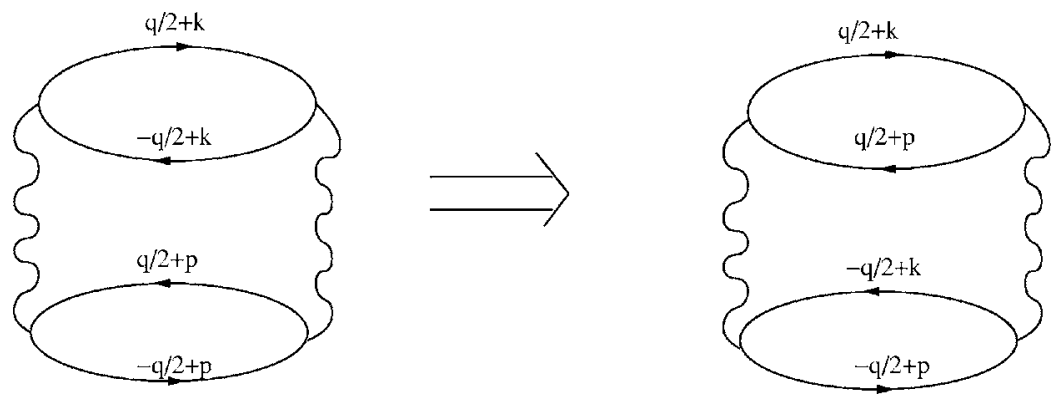

FIG. 1. The diagram for $\Delta \Phi_{2 k_{F}}$, and the trick to compute it. As the nonanalytic part of $\Delta \Phi_{2 k_{F}}$ comes from small $k$ and $p$, it can be reexpressed as a product of two bubbles $\Pi^{\uparrow \downarrow}\left(q^{\prime}, \omega\right)$ with small momentum transfer $\vec{q}^{\prime}=\vec{k}-\vec{p}$.

specific heat. This can be easily understood as the nonanalytic term in the zero field spin susceptibility $\Delta \chi_{s}(T)$ describes a singular response to an infinitesimally small magnetic field. A magnetic field splits the Fermi momentum $k_{F}$ into $k_{F}^{\uparrow}$ and $k_{F}^{\downarrow}$. The small $q$ form of $\Pi^{\uparrow \uparrow}\left(\vec{q}, \Omega_{n}, T\right)$ and $\Pi^{\downarrow \downarrow}\left(\vec{q}, \Omega_{n}, T\right)$ is unaffected by this splitting, up to terms of order $\left(\mu_{B} H / E_{F}\right)^{2}$, hence the response to the infinitesimal field must be analytic in $T$. At the same time, the singular $2 k_{F}$ contribution to $\Phi(T)$ at zero field originates from the fact that the two polarization operators in Eq. (1) are nonanalytic at the same $q=2 k_{F}$. In a field the singularities in the spin-up and the spin-down polarization operators occur at different $q=2 k_{F}^{\uparrow}$ and $q=2 k_{F}^{\downarrow}$. Accordingly, a magnetic field regularizes the $2 k_{F}$ nonanalyticity in the thermodynamic potential, but for a price that the linear response to the field, i.e., the spin susceptibility $\Delta \chi(T, H=0)$, becomes nonanalytic.

Our goal is to analyze the forms of the susceptibility and the specific heat at a finite $H$, i.e., beyond the linear response theory. We consider the fields for which $\mu_{B} H$ is comparable to $T$, but still $\mu_{B} H \ll E_{F}$. For these fields, the nonanalytic contribution to $\Phi$ from small $q$ are unaffected by the field . However the $2 k_{F}$ contribution is field dependent and evolves at $\mu_{B} H \sim T$.

The calculation of $\Delta \Phi=\Phi-\Phi_{0}$ is somewhat tricky. In principle, all one has to do is to evaluate the particle-hole bubbles for fermions with up and down spins at a finite $T$, substitute the results into Eq. (1), integrate over momentum $q$ and sum over Matsubara frequencies $\Omega_{n}$. In practice, however, this computation is easy to perform only for the small $q$ part as for $q \ll k_{F}$, the nonanalytic part of the polarization bubble is associated with the Landau damping, which does not depend on $T$, apart from regular $\left(T / E_{F}\right)^{2}$ corrections. Accordingly, one can safely use the known analytical forms of $\Pi(q, \Omega)$ at $T=0$. For $q$ near $2 k_{F}$, nonanalytic terms in $\Pi^{\uparrow \uparrow}\left(\vec{q}, \Omega_{n}, T\right)$ and $\Pi^{\downarrow \downarrow}\left(\vec{q}, \Omega_{n}, T\right)$ contain scaling functions of $T / \omega$, which are only available in integral forms. ${ }^{7}$ This substantially complicates direct calculation of the $2 k_{F}$ term. There exists, however, a way to compute the $2 k_{F}$ term, which avoids dealing with the $2 k_{F}$ polarization bubbles at a finite $T$. This method explores the fact that only the nonanalytic parts of $\Pi^{\uparrow \uparrow}\left(\vec{q}, \Omega_{n}, T\right)$ and $\Pi^{\downarrow \downarrow}\left(\vec{q}, \Omega_{n}, T\right)$ for $q$ near $2 k_{F}$ contribute to the nonanalyticity in the thermodynamic potential. Earlier works have demonstrated that the $2 k_{F}$ nonanalyticity in $\Pi\left(\vec{q}, \Omega_{n}, T\right)$ comes from fermions in the particle-hole bubble with momenta near $\pm \vec{q} / 2$. $^{6,12}$ This implies that, out of four fermions in the two-bubble second order diagram for the thermodynamic potential in Fig. 1, two fermions with opposite spins have momenta near $\vec{q} / 2$, while the other two fer- mions have momenta near $-\vec{q} / 2$. Then the $2 k_{F}$ part of the $\Delta \Phi$ can be rewritten as the integral over small $\vec{k}$ and small $\vec{p}$ of

$$
\begin{aligned}
\Delta \Phi_{2 k_{F}}= & -\frac{U^{2}}{2} \sum_{\omega_{m}, \omega_{m}^{\prime}, \omega_{m}^{\prime \prime}} \int d^{2} q \int d^{2} k d^{2} p \\
& \times G^{\uparrow}\left(\vec{q} / 2+\vec{k}, \omega_{m}+\omega_{m}^{\prime}\right) G^{\downarrow}\left(\vec{q} / 2+\vec{p}, \omega_{m}^{\prime}\right) \\
& \times G^{\uparrow}\left(-\vec{q} / 2+\vec{k}, \omega_{m}+\omega_{m}^{\prime \prime}\right) G^{\downarrow}\left(-\vec{q} / 2+\vec{p}, \omega_{m}^{\prime \prime}\right)
\end{aligned}
$$

or, equivalently, as

$$
\Delta \Phi_{2 k_{F}}=-\frac{U^{2}}{2} \sum_{n} \int \frac{d^{d} q^{\prime}}{(2 \pi)^{d}}\left[\Pi^{\uparrow \downarrow}\left(\vec{q}^{\prime}, \Omega_{n}, T\right)\right]^{2},
$$

where the integration is confined to small $\vec{q}^{\prime}=\vec{k}-\vec{p}$. In other words, the nonanalytic $2 k_{F}$ contribution to the thermodynamic potential can be reexpressed in terms of the particlehole bubble for fermions with opposite spins and a small momentum transfer. The non-analytic term in $\Pi$ at small $\vec{q}^{\prime}$ does not depend on temperature (apart from irrelevant corrections), hence $\Pi^{\uparrow \downarrow}\left(\vec{q}^{\prime}, \Omega_{n}, T\right)$ can be safely approximated by $\Pi^{\uparrow \downarrow}\left(\vec{q}^{\prime}, \Omega_{n}, 0\right)$. At the same time, the polarization bubble $\Pi^{\uparrow} \downarrow\left(\vec{q}, \Omega_{n}, 0\right)$ strongly depends on the magnetic field [contrary to $\left.\Pi^{\uparrow \uparrow}\left(\vec{q}, \Omega_{n}, 0\right)\right]$, and this gives rise to the scaling dependence on $\mu_{B} H / T$.

Combining the $q=0$ and $2 k_{F}$ contributions, we obtain for the thermodynamic potential

$$
\begin{aligned}
\Delta \Phi= & -\frac{U^{2}}{2} \sum_{n} \int \frac{d^{d} q}{(2 \pi)^{d}}\left[\left(\Pi^{\uparrow \downarrow}\left(\vec{q}, \Omega_{n}, 0\right)\right)^{2}\right. \\
& \left.+\Pi^{\uparrow \uparrow}\left(\vec{q}, \Omega_{n}, 0\right) \Pi^{\downarrow \downarrow}\left(\vec{q}, \Omega_{n}, 0\right)\right],
\end{aligned}
$$

where the integration now involves only small $q$. We now proceed separately with the $2 \mathrm{D}$ and $3 \mathrm{D}$ cases.

\section{2D CASE}

In $2 \mathrm{D}$ we have

$$
\begin{gathered}
\Pi^{\uparrow} \downarrow\left(\vec{q}, \Omega_{n}, T\right)=\frac{m}{2 \pi} \frac{\Omega_{n}}{\sqrt{\left(\Omega_{n}-i \delta \mu\right)^{2}+\left(v_{F} q\right)^{2}}}+\cdots \\
\Pi^{\uparrow \uparrow}\left(\vec{q}, \Omega_{n}, T\right)=\frac{m}{2 \pi} \frac{\Omega_{n}}{\sqrt{\Omega_{n}^{2}+\left(v_{F} q\right)^{2}}}+\cdots,
\end{gathered}
$$

where dots stand for analytic terms, expandable in powers of $\Omega_{n}^{2}$ or $q^{2}$, and $\delta \mu=\mu_{\uparrow}-\mu_{\downarrow}=2 \mu_{B} H$. Substituting Eq. (5) into 


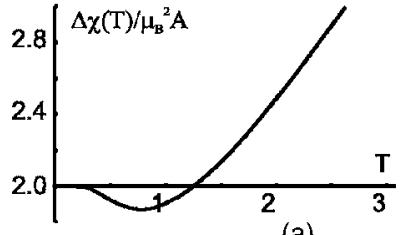

(a)

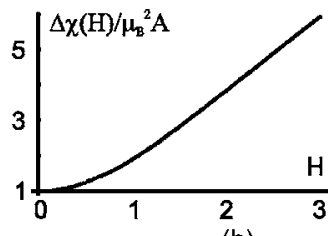

(b)
FIG. 2. $\Delta \chi$ as a function of temperature at fixed magnetic field (a) and as a function of magnetic field at fixed temperature (b). In the left panel, $T$ and $\Delta \chi / \mu_{B}^{2} A$ are in units of $\mu_{B} H$ ( $A$ is defined in the text). In the right panel $\mu_{B} H$ and $\Delta \chi / \mu_{B}^{2} A$ are in units of $T$. In these units, $\Delta \chi / \mu_{B}^{2} A=f_{\chi}\left(\mu_{B} H\right)$.

Eq. (4) and integrating over momentum $q$ we obtain

$$
\Delta \Phi=\left(\frac{m}{2 \pi}\right)^{2} \frac{U^{2} T}{8 \pi v_{F}^{2}} \sum_{n} \Omega_{n}^{2} \ln \left[\frac{\left(\Omega_{n}-2 i \mu_{B} H\right)^{2} \Omega_{n}^{2}}{E_{F}^{4}}\right] .
$$

Differentiating Eq. (6) with respect to $H$, we obtain

$$
\Delta M=-\frac{\partial \Delta \Phi}{\partial H}=\frac{\mu_{B}{ }^{4} m^{4} U^{2} H^{3}}{\pi^{3} k_{F}^{2}} T \sum_{n} \frac{1}{\Omega_{n}{ }^{2}+\left(2 \mu_{B} H\right)^{2}} .
$$

Summation over Matsubara frequencies gives

$$
\Delta M=\mu_{B} A T^{2} x^{2}\left[1+2 n_{B}(2 x)\right],
$$

where

$$
A=\frac{m^{4} U^{2}}{4 \pi^{3} k_{F}^{2}}, \quad x=\mu_{B} H / T .
$$

We see from Eq. (7) that $\Delta M$ increases in a field due to two reasons. First, the field leads to a finite magnetization at $T$ $=0$, and second, a finite field populates the system with spin waves precessing at the energy $\mu_{B} H$. Differentiating Eq. (7) again over $H$, we obtain the spin susceptibility in the form

$$
\Delta \chi(T, H)=\chi(T, H)-\chi(0,0)=\mu_{B}^{2} A T f_{\chi}(x),
$$

where

$$
f_{\chi}(x)=\frac{x}{\sinh ^{2}(x)}[\sinh (2 x)-x] .
$$

For vanishing $H$, i.e., at $x \rightarrow 0, f_{\chi}(0)=1$, and

$$
\Delta \chi(T, H)=\chi(T, 0)-\chi(0,0)=\mu_{B}^{2} A T .
$$

This coincides with the earlier result. ${ }^{10}$ In the opposite limit of large $x, f_{\chi}(x) \approx 2 x$, and

$$
\Delta \chi(T, H)=2 \mu_{B}^{2} A T|x|=2 \mu_{B}^{3} A|H| .
$$

We see that at high fields, the spin susceptibility scales as $|H|$, i.e., is nonanalytic in $H$.

In Fig. 2 we plot the susceptibility as a function of temperature at a given $H$, and as a function of the magnetic field at a given $T$. Note that at a finite $H$, the Bose term in Eq. (7) gives rise to a negative derivative of $\partial \Delta \chi / \partial T$. This in turn gives rise to a shallow minimum in the temperature dependence of $\Delta \chi(T, H)$.

The specific heat $\Delta \gamma(T, H)=\gamma(T, H)-\gamma(0,0)$ is obtained by differentiating Eq. (6) twice over $T$ (see Fig. 3). At $H$

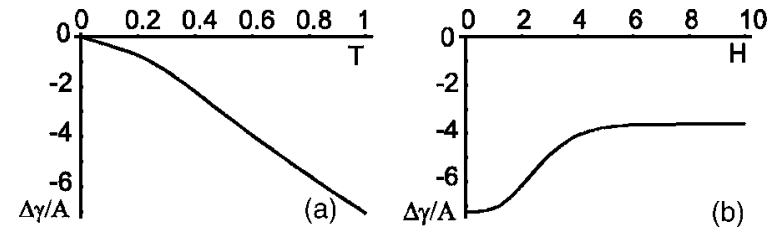

FIG. 3. $\Delta \gamma$ as a function of temperature at fixed magnetic field (a) and as a function of magnetic field at fixed temperature (b). In the left panel, $T$ and $\Delta \chi / \mu_{B}^{2} A$ are in units of $\mu_{B} H$. In the right panel $\mu_{B} H$ and $\Delta \chi / \mu_{B}^{2} A$ are in units of $T$. In these units $\Delta \gamma / A$ $=f_{\gamma}\left(\mu_{B} H\right)$.

$=0, \Delta \gamma(T, H)=-6 A T \zeta(3) \cdot{ }^{10,12,13}$ At a finite $H$,

$$
\Delta \gamma(T, H)=-A T f_{\gamma}(x),
$$

where

$$
\begin{aligned}
f_{\gamma}(x)= & 3\left[\operatorname{Li}_{3}\left(e^{-2 x}\right)+2 x \operatorname{Li}_{2}\left(e^{-2 x}\right)-2 x^{2} \ln \left(1-e^{-2 x}\right)\right] \\
& +6 \zeta(3)-2 x^{3}+4 x^{3} \operatorname{coth} x-x^{3} \frac{1}{\sinh ^{2} x}(\sinh 2 x-x)
\end{aligned}
$$

and $L i$ are polylogarithmic functions. At $x \ll 1, f_{\gamma}(x)$ $\approx 6 \zeta(3)-x^{4} / 6$ and

$$
\Delta \gamma\left(\frac{\mu_{B} H}{T} \ll 1\right) \approx-A T\left[6 \zeta(3)-\frac{1}{6}\left(\frac{\mu_{B} H}{T}\right)^{4}\right] .
$$

In the opposite limit of $x \gg 1, f_{\gamma}(x)=3 \zeta(3)+4 x^{4} e^{-2 x}$, and

$$
\Delta \gamma\left(\frac{\mu_{B} H}{T} \gg 1\right) \approx-A T\left[3 \zeta(3)+4\left(\frac{\mu_{B} H}{T}\right)^{4} e^{-2 \mu_{B} H / T}\right] .
$$

In both limits the temperature dependence of the specific heat is linear in $T$, but the prefactor changes by a factor of 2 between small and high fields. This result could be anticipated as a high magnetic field eliminates the non-analyticity in the polarization bubble $\Pi^{\uparrow} \downarrow\left(\vec{q}, \Omega_{n},\right)$, such that only the second term in Eq. (4) contributes to the $T$ term in $\Delta \gamma(T, H)$.

The above results can be straightforwardly extended to an arbitrary $U(q)$. For the susceptibility, the prefactor in Eq. (9), contains $U\left(2 k_{F}\right)$ instead of $U \cdot{ }^{6,7,10}$ For the specific heat coefficient, we have, instead of Eq. (9)

$$
\begin{aligned}
\Delta \gamma(T, H)= & -\frac{3 \zeta(3) m^{4}}{2 \pi^{3} k_{F}^{2}} T\left[\left(U(0)-\frac{1}{2} U\left(2 k_{F}\right)\right)^{2}\right. \\
& +\frac{U^{2}\left(2 k_{F}\right)}{4}\left(1+2 \frac{f_{\gamma}(x)-3 \zeta(3)}{3 \zeta(3)}\right) .
\end{aligned}
$$

The combinations $U(0)-1 / 2 U\left(2 k_{F}\right)$ and $-1 / 2 U\left(2 k_{F}\right)$ are the charge and spin components of the scattering amplitude at the scattering angle $\pi, A_{c}(\pi)$, and $A_{s}(\pi)$, respectively. At large $x, f_{\gamma}(x) \approx 3 \zeta(3)$, and the last term in the right-hand side (RHS) of Eq. (14) vanishes. This implies that at a large field, only the longitudinal component of $A_{s}$ contributes to $\Delta \gamma(T)$.

\section{3D CASE}

We now consider the $3 \mathrm{D}$ case. in $3 \mathrm{D}$, the polarization operators at small $q$ are given by $^{2}$ 


$$
\begin{gathered}
\Pi^{\uparrow \downarrow}\left(\vec{q}, \Omega_{n}, 0\right)=\frac{m k_{F}}{2 \pi^{2}} \frac{\Omega_{n}}{v_{F} q} \arctan \frac{\left(\Omega_{n}-i \delta \mu\right)}{v_{F} q}+\cdots, \\
\Pi^{\uparrow \uparrow}\left(\vec{q}, \Omega_{n}, T\right)=\frac{m k_{F}}{2 \pi^{2}} \frac{\Omega_{n}}{v_{F} q} \arctan \frac{\Omega_{n}}{v_{F} q}+\cdots .
\end{gathered}
$$

As before, the ellipsis stand for the analytic terms which are expandable in powers of $\Omega_{n}^{2}$ or $q^{2}$, and $\delta \mu=\mu_{\uparrow}-\mu_{\downarrow}=2 \mu_{B} H$. Differentiating the thermodynamic potential, (4) with respect to $H$, we obtain the corrections to the magnetization

$$
\Delta M=-\frac{\partial \Delta \Phi}{\partial H}=-\frac{\mu_{B}\left(m U k_{F}\right)^{2}}{4 \pi^{5} v_{F}^{3}} T \sum_{n} \Omega_{n}{ }^{2} \arctan \frac{2 \mu_{B} H}{\left|\Omega_{n}\right|} .
$$

Differentiating further with respect to $H$, we obtain

$$
\begin{aligned}
\Delta \chi_{s}(T, H)= & -\frac{\mu_{B}^{2}\left(m U k_{F}\right)^{2}}{2 \pi^{5} v_{F}^{3}} \\
& \times\left[T \sum_{n=1}^{M} \Omega_{n}+4\left(\mu_{B} H\right)^{2} T \sum_{n=1}^{M} \frac{\Omega_{n}}{\Omega_{n}^{2}+4 \mu_{B}^{2} H^{2}}\right],
\end{aligned}
$$

where $M \sim E_{F} / T$ is the upper cutoff in the summation over frequency. The first term in the RHS of Eq. (16) is the susceptibility at zero field. By power counting, one might expect the spin susceptibility $\chi_{s}(T)$ in 3D to scale as $T^{2} \ln T$. However, the Matsubara sum $T \sum_{n=1}^{M} \Omega_{n}$ only contains a $T$-independent term, of order $E_{F}^{2}$, and the term -(1/6) $\pi T^{2}$. This last term is universal in the sense that it comes from fermions close to the Fermi surface, but it is analytic in $T$. As a result, $\Delta \chi_{s}(T, 0) \propto T^{2}$ is also analytic and not important as the analytic in $T$ contributions to $\chi_{s}(T)$ are already present in the Lindhard function for free fermions. The absence of the nonanalytic temperature correction to the spin susceptibility in 3D was first noticed in Ref. 6 (see also Ref. 5). The second term in the RHS of Eq. (16) is the extra contribution in a finite field. Evaluating the Matsubara sum we find that this contribution scales as $H^{2} \ln \left\{\max \left(T, \mu_{B} H\right)\right\}$. We see therefore that in a finite magnetic field, $\chi_{s}(T)$ does indeed become nonanalytic. Casting $\Delta \chi_{s}(T, H)$ into the scaling form, we obtain

$$
\Delta \chi_{s}(T, H)=\chi_{0}\left(\frac{m U k_{F}}{2 \pi^{2}}\right)^{2}\left(\frac{T}{E_{F}}\right)^{2} g\left(\frac{\mu_{B} H}{T}\right),
$$

where $\chi_{0}=\mu_{B}^{2} k_{F}^{3} /\left(2 \pi^{2} E_{F}\right)$ is Pauli susceptibility, and to a logarithmic accuracy

$$
g(x)=x^{2} \ln \left[\frac{E_{F}}{T \times \max \{x, 1\}}\right] .
$$

The $H^{2} \ln H$ dependence of $\chi(H)$ was earlier reported by Misawa. ${ }^{19}$ However, his prefactor is different from the one we obtained.

Differentiating the thermodynamic potential twice over $T$, we also obtain the field dependence of the specific heat co- efficient. This field dependence in 3D parallels the one for 2D systems. Namely, at zero field,

$$
\begin{aligned}
\Delta \gamma(T, 0) & \\
= & -\frac{3}{20} \frac{\left(m k_{F}\right)^{2}}{\pi^{2}}\left[\left(U(0)-\frac{1}{2} U\left(2 k_{F}\right)\right)^{2}+\frac{3}{4} U^{2}\left(2 k_{F}\right)\right] \\
& \times\left(\frac{T}{E_{F}}\right)^{2} \ln \frac{E_{F}}{T} .
\end{aligned}
$$

In a finite field, the charge part is not affected, while in the spin part, the $\operatorname{logarithmic}$ factor $3 \ln \left(E_{F} / T\right)$ is replaced by $\ln \left(E_{F} / T\right)+2 \ln \left(E_{F} / \max \left\{T, \mu_{B} H\right\}\right)$. As a result, at $\mu_{B} H \gg T$, $\Delta \gamma(T, H)$ still behaves as $T^{2} \ln T$, with a smaller prefactor.

\section{AN EXTENSION TO ARBITRARY $U(q)$}

The scaling forms for the susceptibility and the specific heat, Eqs. (13), (14), (17), and (19) are obtained to second order in the interaction $U(q)$. For 2D systems without a field, it has been demonstrated ${ }^{12,18}$ that the second-order results, reexpressed via the spin and charge components of the scattering amplitude $A(\pi)$, are actually the exact Fermi-liquid expressions, valid at arbitrary strong $U(q)$. The formal procedure is to replace $U(0)$ by $A_{c}(\pi)-A_{s}(\pi)$, and replace $U\left(2 k_{F}\right)-2 A_{s}(\pi)$. We found that the same remains true in the presence of the field, i.e., our scaling functions are exact, once $U(0)$ and $U\left(2 k_{F}\right)$ are reexpressed in terms of $A(\pi)$.

In $3 \mathrm{D}$ the situation is more involved as there are two physically distinct nonanalytic contributions, from secondorder perturbation theory, and from third-order perturbation theory ${ }^{20}$ The third-order terms lead to the same nonanalytic behavior at $H=0$ as second-order terms ${ }^{3}$ and likely give rise to the same field dependencies as we found to order $U^{2}$. Still, the full results for $\Delta \chi_{s}(T, H)$ and $\Delta \gamma(T, H)$ in 3D cannot be obtained by a simple extension of the second-order formulas.

\section{SUMMARY}

To summarize, in this paper we analyzed in perturbation theory nonanalytic terms in the magnetization, the spin susceptibility and the specific heat of 2D and 3D Fermi liquids, in an external magnetic field $\mu_{B} H \ll E_{F}$. We obtained the nonanalytic terms in the forms of scaling functions of $\mu_{B} H / T$. We found that at $\mu_{B} H \gg T$, the spin susceptibility scales as $|H|$ in $2 \mathrm{D}$ and as $H^{2} \ln |H|$ in $3 \mathrm{D}$. The specific heat in a field preserves the same temperature dependence as in the absence of a field, but the prefactor changes between small and large values of $\mu_{B} H / T$.

\section{ACKNOWLEDGMENTS}

We thank D. Belitz, P. Fulde, D. Maslov, and T. Vojta for useful discussions. The research has been supported by NSF Grant No. DMR 0240238 (A.Ch.), and the Guest Scientists Program of the Max Planck Society (J.B.). 
${ }^{1}$ L. D. Landau Sov. Phys. JETP 3, 920 (1956); 5, 101 (1957); 8, 70 (1958)

${ }^{2}$ A. A. Abrikosov, L. P. Gorkov, and I. E. Dzyaloshinski, Methods of Quantum Field Theory in Statistical Physics (Dover Publications, New York, 1963).

${ }^{3}$ S. Doniach and S. Engelsberg, Phys. Rev. Lett. 17, 750 (1966); W. F. Brinkman and S. Engelsberg, Phys. Rev. 169417 (1968); A. I. Larkin and V. I. Mel'nikov, Sov. Phys. JETP, 20, 173 (1975); M. T. Beal-Monod, S.-K. Ma, and D. R. Fredkin,Phys. Rev. Lett. 20, 929 (1968).

${ }^{4}$ D. J. Amit, J. W. Kane, and H. Wagner, Phys. Rev. 175, 313 (1968); 175, 326 (1968).

${ }^{5}$ G. M. Carneiro and C. J. Pethick, Phys. Rev. B 16, 1933 (1977).

${ }^{6}$ D. Belitz, T. R. Kirkpatrick, and T. Vojta, Phys. Rev. B 55, 9452 (1997).

${ }^{7}$ G. Y. Chitov and A. J. Millis, Phys. Rev. Lett. 865337 (2001); Phys. Rev. B 64, 054414 (2001).

${ }^{8}$ D. Coffey and K. S. Bedell, Phys. Rev. Lett. 71, 1043 (1993)

${ }^{9}$ M. A. Baranov, M. Yu. Kagan, and M. S. Mar'enko, JETP Lett. 58, 709 (1993)

${ }^{10}$ A. V. Chubukov and D. L. Maslov, Phys. Rev. B 68, 155113 (2003).

${ }^{11}$ V. M. Galitski and S. Das Sarma, Phys. Rev. B 70, 035111
(2004).

${ }^{12}$ A. V. Chubukov, D. L. Maslov, S. Gangadharaiah, and L. I. Glazman, cond-mat/0412283 (unpublished).

${ }^{13}$ G. Catelani, I. L. Aleiner, JETP 100, 331 (2005).

${ }^{14}$ V. M. Galitski, A. V. Chubukov, and S. Das Sarma, cond-mat/ 0501132 (unpublished).

${ }^{15}$ See, e.g., D. S. Greywall, Phys. Rev. B 41, 1842 (1990); A. Casey, H. Patel, J. Nyeki, B. P. Cowan, and J. Saunders, Phys. Rev. Lett. 90, 115301 (2003).

${ }^{16}$ J. Zhu et al., Phys. Rev. Lett. 90, 056805 (2003).

${ }^{17}$ O. Prus, Y. Yaish, M. Reznikov, U. Sivan, and V. Pudalov, Phys. Rev. B 67, 205407 (2003); A. A. Shashkin, M. Rahimi, S. Anissimova, S. V. Kravchenko, V. T. Dolgopolov, and T. M. Klapwijk, Phys. Rev. Lett. 91, 046403 (2003); V. M. Pudalov, M. E. Gershenson, H. Kojima, N. Butch, E. M. Dizhur, G. Brunthaler, A. Prinz, and G. Bauer, ibid. 88, 196404 (2002); J. Zhu, H. L. Stormer, L. N. Pfeiffer, K. W. Baldwin, and K. W. West, ibid. 90, 056805 (2003).

${ }^{18}$ Andrey V. Chubukov, Dmitrii L. Maslov, Suhas Gangadharaiah, and Leonid I. Glazman, Phys. Rev. Lett. 95, 026402 (2005)

${ }^{19}$ S. Misawa, Phys. Rev. Lett. 26, 1632 (1971).

${ }^{20}$ C. J. Pethick and G. M. Carneiro, Phys. Rev. A 7, 304 (1973). 